\title{
The malaria parasite Plasmodium vivax exhibits greater genetic diversity than Plasmodium falciparum
}

\author{
Daniel E Neafsey ${ }^{1}$, Kevin Galinsky ${ }^{1}$, Rays H Y Jiang ${ }^{1}$, Lauren Young ${ }^{1}$, Sean M Sykes ${ }^{1}$, Sakina Saif ${ }^{1}$, \\ Sharvari Gujja ${ }^{1}$, Jonathan M Goldberg ${ }^{1}$, Sarah Young ${ }^{1}$, Qiandong Zeng ${ }^{1}$, Sinéad B Chapman ${ }^{1}$, Aditya P Dash ${ }^{2,6}$, \\ Anupkumar R Anvikar ${ }^{2}$, Patrick L Sutton ${ }^{3}$, Bruce W Birren ${ }^{1}$, Ananias A Escalante ${ }^{4}$, John W Barnwell ${ }^{5}$ \& \\ Jane M Carlton ${ }^{3}$
}

\begin{abstract}
We sequenced and annotated the genomes of four $P$. vivax strains collected from disparate geographic locations, tripling the number of genome sequences available for this understudied parasite and providing the first genome-wide perspective of global variability in this species. We observe approximately twice as much SNP diversity among these isolates as we do among a comparable collection of isolates of P. falciparum, a malaria-causing parasite that results in higher mortality. This indicates a distinct history of global colonization and/or a more stable demographic history for $P$. vivax relative to $P$. falciparum, which is thought to have undergone a recent population bottleneck. The SNP diversity, as well as additional microsatellite and gene family variability, suggests a capacity for greater functional variation in the global population of $P$. vivax. These findings warrant a deeper survey of variation in $P$. vivax to equip disease interventions targeting the distinctive biology of this neglected but major pathogen.
\end{abstract}

Half the world's population is estimated to be at risk for malaria caused by $P$. vivax ${ }^{1}$, owing to this parasite's unique potential for lengthy remission and tolerance of cooler climates than those preferred by strictly tropical Plasmodium species. Although the P. falciparum parasite is responsible for the majority of contemporary malariarelated mortality, there is evidence that $P$. vivax may have been a more virulent parasite before the advent of modern medicine. As far north as England, death records indicate that $P$. vivax likely reduced the average lifespan from 58 to 33 years during the nineteenth century ${ }^{2}$. More recently, studies have shown that $P$. vivax is capable of causing the severe malaria syndromes that have long been attributed only to P. falciparum ${ }^{3}$.

In spite of the past and present impact of $P$. vivax on human health, it remains chronically understudied relative to $P$. falciparum. The ability to continuously culture $P$. falciparum but not $P$. vivax in the laboratory, compounded with the differential mortality imposed by the two species, has led to vast discrepancies in the state of knowledge regarding almost all aspects of the biology of these species. The recently renewed push for malaria eradication may remain incomplete unless this disparity in knowledge is addressed and the findings of new studies applied to disease control programs ${ }^{4}$.

Genetic diversity is important to characterize in order to understand the history of human association with a disease, to evaluate the direct effects of diversity on clinical disease and also because it may directly or indirectly reduce the efficacy of therapeutics such as drugs and vaccines. In contrast to P. falciparum, for which the genomes of many hundreds of isolates have now been sequenced or genotyped $^{5}$, only the P. vivax genomic reference strain (Salvador I) ${ }^{6}$ and unassembled shotgun sequencing of a Peruvian isolate (IQ07) ${ }^{7}$ have been completed. We sequenced, assembled and annotated the genomes of four geographically disparate isolates of $P$. vivax to remedy the lack of genetic diversity data available for this species relative to P. falciparum. The designations and geographic origins of each P. vivax strain are given in Table 1, which also lists the names and origins of a concurrently sequenced comparator panel of $P$. falciparum isolates that came from similarly disparate geographic locales. Templates of both species were sequenced using the same next-generation sequencing platform (Illumina Genome Analyzer IIx and HiSeq 2000) and evaluated for diversity using the same bioinformatic tools. All four of the newly sequenced P. vivax reference strains (North Korean, India VII, Mauritania I and Brazil I) are clonal infections adapted for growth in monkeys, are publically available via the Malaria Research and Reference Reagent Resource Center (MR4; see URLs) and were sequenced from genomic DNA derived from leukocytedepleted monkey blood (Online Methods). The P. falciparum isolates (from Honduras, India, Indochina (Thailand and Laos) and Senegal) were also clonal and were sequenced using template derived from in vitro cultures. We generated de novo assemblies for each of the four P. vivax isolates (Table 2) using the ALLPATHS LG assembly algorithm ${ }^{8}$. Assembly quality was substantially higher for $P$. vivax

\footnotetext{
${ }^{1}$ Broad Institute, Cambridge, Massachusetts, USA. ${ }^{2}$ National Institute of Malaria Research, Indian Council of Medical Research, Dwarka, New Delhi, India.

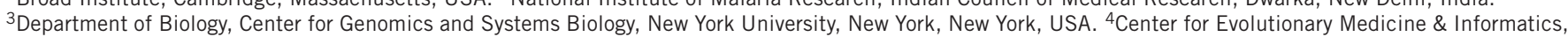
The Biodesign Institute, School of Life Sciences, Arizona State University, Tempe, Arizona, USA. ${ }^{5}$ Center for Global Health, Centers for Disease Control and Prevention, Division of Parasitic Diseases and Malaria, Atlanta, Georgia, USA. ${ }^{6}$ Present address: World Health House, New Delhi, India. Correspondence should be addressed to J.M.C. (jane.carlton@nyu.edu).
} 
Table 1 Strains and isolates of $P$. vivax and $P$. falciparum used in this study

\begin{tabular}{lll}
\hline Geographic origin & \multicolumn{1}{c}{ P. vivax strains and isolates } & \multicolumn{1}{c}{ P. falciparum strains } \\
\hline Latin America & $\begin{array}{l}\text { Salvador I (El Salvador) (ref. 6) } \\
\text { Brazil I (ref. 23) }\end{array}$ & HB3 (Honduras) (ref. 24) \\
& IQ07 (Peru) (ref. 7) & \\
South Asia (India) & India VII (ref. 25) & ML-14 \\
& & $87 \_239$ \\
East Asia & North Korean (ref. 26) & Dd2 (Indochina: Thailand \\
Africa & Mauritania I (ref. 28) & and Laos) (ref. 27) \\
& & 3D7 (ref. 29) \\
& & Th231.08 (Senegal) (ref. 30)
\end{tabular}

Information in parentheses give more specific geographic origins, where known.

than for a $P$. falciparum assembly generated using the same approach (Supplementary Table 1), likely due to the more moderate AT nucleotide composition of the P. vivax genome ( $P$. vivax: $57.7 \%$ AT versus P. falciparum: $80.6 \%$ AT). Synteny between the new $P$. vivax assemblies and the Salvador I reference assembly was found to be highly conserved ( $\geq 97.9 \%$; Supplementary Fig. 1).

We next used the sequencing data to evaluate genetic diversity within each species. The pairwise SNP rate for each sequenced isolate relative to the reference assembly for each species ( $P$. vivax, Salvador I; $P$. falciparum, 3D7) is given in Figure 1a as a function of inferred SNP quality. Pairwise SNP rate relative to a reference assembly is expected to be a function of evolutionary or geographic distance, but, despite the varying geographic origin of isolates from both species, we found that the $P$. vivax SNP rates were uniformly higher than the $P$. falciparum SNP rates, regardless of the SNP quality threshold used. This finding suggests globally higher genetic diversity in $P$. vivax relative to $P$. falciparum, a genome-wide result confirming previous pilot surveys $^{9,10}$. We explored this finding by comparing mean SNP rates according to sequence class, using SNPs with a minimum PHREDstyle quality score of 30 (estimated accuracy of at least $99.9 \%)^{11}$. We found that $P$. vivax exhibited a significantly higher mean SNP rate than $P$. falciparum at intergenic and intronic fourfold-degenerate synonymous coding sites, within coding sequences overall and across all sequence classes ( $t$ test, $P=0.0087$; Fig. 1b), suggesting that the difference in diversity is pervasive and occurs across the genome. To control for variation in the degree of functional constraint among genes within each genome, we next evaluated mean pairwise SNP diversity $(\pi)$ in a collection of 3,401 genes for which we could confidently identify $1: 1$ orthologs in the two species using the reciprocal best BLAST hits $(\mathrm{RBH})$ criterion. In this comparison, we observed approximately twice as much SNP diversity in $P$. vivax compared to $P$. falciparum (paired $t$ test, $P=2.2 \times 10^{-16}$; Fig. 1c), confirming the ubiquity and magnitude of the SNP diversity disparity between the species (Supplementary Fig. 2 and Supplementary Table 2).

To test whether a differing SNP mutation rate, rather than a different effective population size and/or demographic history, can account for the differences in SNP diversity observed, we compared the genome-wide diversity of microsatellites, a different class of mutation. Because microsatellite length variants are caused by replication slippage rather than point substitution ${ }^{12}$, we would expect their relative diversity profile to be different from that of SNPs under the null hypothesis that a different point mutation rate explains the disparity in SNP diversity. We applied a new method of evaluating microsatellite length variation from Illumina data and observed, as we had with the SNPs, significantly higher diversity in $P$. vivax than in $P$. falciparum (bootstrapping, 279 and 22,713 microsatellites with at least 8 repeats in $P$. vivax and $P$. falciparum, respectively; $P=0.021$; Fig. 1d and Supplementary Fig. 3). Given the population genetic evidence that $P$. falciparum underwent multiple drug-induced selective sweeps and at least one significant bottleneck ${ }^{13}$, these results indicate that $P$. vivax may have a comparatively large effective population size due to an absence of such demographic events in recent history. Even in the face of common drug pressure, $P$. vivax may have disproportionate demographic stability due to its unique capacity for dormancy within infected hosts.

Our sample is smaller than is ideal for evaluating the time to the most recent common ancestor (TMRCA), but by comparing the deepest pairwise nucleotide divergence observed for each species at fourfold-degenerate synonymous sites $(P$. vivax: Mauritania versus Brazil I, $1.628 \times 10^{-3}$ substitutions per site; $P$. falciparum: $3 \mathrm{D} 7$ versus $\mathrm{Dd} 2,9.59 \times 10^{-4}$ substitutions per site) we can predict that the lower bound for TMRCA in P. vivax is approximately $70 \%$ greater than that for $P$. falciparum. The calculation of absolute TMRCA dates is dependent on the accuracy of the inferred mutation rate, and, therefore, results must be interpreted with caution. Nevertheless, if we assume a commonly accepted eukaryotic genome-wide mutation rate for fourfold-degenerate sites $\left(2.2 \times 10^{-9}\right.$ substitutions per site per year ${ }^{14}$, similar to a Plasmodium rate estimated with less precision) $)^{15}$, we can estimate the TMRCA as 768,000 and 452,000 years ago for $P$. vivax and $P$. falciparum, respectively. This $P$. vivax estimate is deeper than previous TMRCA estimates generated using a small number of loci in a larger population sample ${ }^{16}$, but reconciliation of the absolute estimates is difficult without knowledge of the true mutation rate. Assuming mutation rates are similar in both parasite lineages, however, the result stands that $P$. vivax has a much deeper TMRCA.

Other departures in the global population history of these two species are indicated by the topology and branch lengths of their respective phylograms (Fig. 2). The relatively large degree of divergence between the IQ07 Peruvian isolate and the Brazil I and Salvador I strains of $P$. vivax suggests a distinct history in the New World relative to $P$. falciparum, which exhibits low diversity in the New World and is thought to have been introduced within the last 500 years via the African slave trade ${ }^{17}$. The high New World diversity of $P$. vivax, combined with the closer phylogenetic affinity of the three New World $P$. vivax isolates with the east Asian (North Korean) rather than the African (Mauritania I) or south Asian (India VII) strains, could suggest the precolonial arrival of $P$. vivax in the New World accompanying human dispersal from Asia by sea or, less likely, by the Bering land bridge during the last glacial maximum. Alternatively, this profile could be explained by recent but very large-scale (relative to $P$. falciparum) postcolonial introductions to the New World

Table 2 Assembly statistics of four $P$. vivax reference strains sequenced using Illumina technology

\begin{tabular}{|c|c|c|c|c|c|c|c|}
\hline Strain & Assembly size (Mb) & Fold coverage & Contig N50 (kb) & Number of contigs & Scaffold N50 (kb) & Number of scaffolds & $\begin{array}{c}\text { Coverage of Salvador I } \\
\text { reference }(\%)\end{array}$ \\
\hline Brazil I & 28.87 & 68.5 & 28.2 & 1,999 & 885.6 & 260 & 98.0 \\
\hline India VII & 29.25 & 35.0 & 21.2 & 3,358 & 594.6 & 568 & 98.1 \\
\hline Mauritania I & 28.43 & 91.1 & 39.4 & 1,510 & 945.1 & 205 & 97.9 \\
\hline North Korean & 29.65 & 87.6 & 22.1 & 2,499 & 317.6 & 541 & 98.8 \\
\hline
\end{tabular}


Figure 1 Disparity in SNP and microsatellite diversity between $P$. vivax and $P$. falciparum. (a) Quality score versus pairwise whole-genome SNP divergence rates compared to reference assemblies. Blue lines represent $P$. vivax isolates, and red lines represent $P$. falciparum isolates. (b) $P$. falciparum (red shades) versus $P$. vivax (blue shades) Q30 call rates for: coding sequences (CDS), whole-genome, 3' flanking sequence, $5^{\prime}$ flanking sequence, all intergenic sequences, introns and fourfold-degenerate synonymous coding sites (4D). (c) Density distribution of $P$. falciparum-P. vivax diversity log ratios for genes with $1: 1$ orthologs compared to the null expected distribution centered on 1. (d) Histogram of microsatellite diversity in microsatellite loci with a repeat unit size of $2 \mathrm{bp}$. Error bars indicate standard errors. Asterisks indicate size bins for which $P$. vivax is significantly more diverse than $P$. falciparum (bootstrapping, $P<0.05$ ).

via transoceanic trade from east Asia, the Pacific or Europe, the last of which presumably harbored a genetically distinct parasite clade before disease elimination ${ }^{18}$. Deeper genomic sampling of $P$. vivax populations will be required to explain these patterns in the diversity data.

We next explored the profile of variation in individual genes and gene families to evaluate the potential functional consequences of the extremely high genomic diversity we observe in $P$. vivax. Mean pairwise divergence among the sequenced $P$. vivax isolates is highest in gene families associated with red blood cell invasion and immune evasion (Fig. 3a). Functional enrichment analysis of diversity in individual genes also found nonsynonymous SNPs to be concentrated in invasion-related motility genes (Supplementary Table 3). This extremely high sequence diversity suggests that vaccines targeting polymorphic antigens may encounter an even greater hurdle in eliciting cross-protective immune responses than they do in P. falciparum, where strain-specific immunity has been recently observed to limit vaccine efficacy ${ }^{19}$.

Differences in the distribution of nonsynonymous SNPs among genes with orthologs in both species are potentially reflective of differences in disease biology in $P$. vivax and $P$. falciparum (Supplementary Table 4). Genes expressed in the pre-erythrocytic stages are the most enriched group among those with higher ratios of nonsynonymousto-synonymous diversity $\left(\pi_{\mathrm{NS}} / \pi_{\mathrm{S}}\right)$ in $P$. vivax relative to $P$. falciparum (Mann-Whitney $U$ test; $Z$ score $=2.2$ ), whereas genes associated with

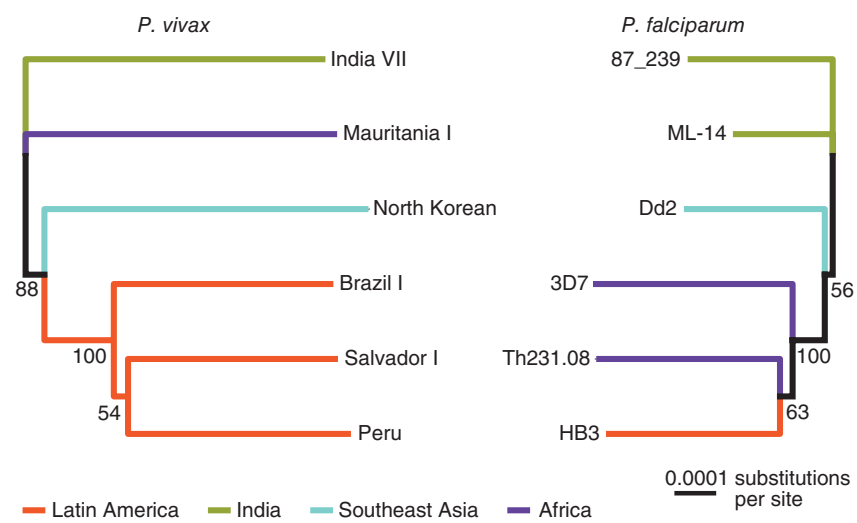

b

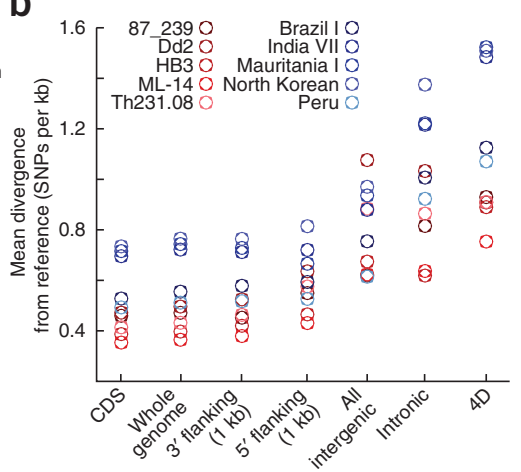

d

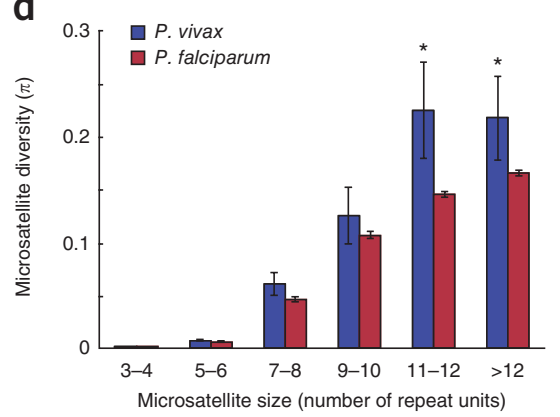

host-parasite interactions are the most enriched group with higher $\pi_{\mathrm{NS}} / \pi_{\mathrm{S}}$ in $P$. falciparum relative to $P$. vivax (Mann-Whitney $U$ test; $Z$ score $=2.4)($ Supplementary Table 5$)$. Although neither of these enrichments was statistically significant after correction for multiple testing, this pattern bears further exploration when sequence data from more $P$. vivax genomes become available.

As expected, we observed enormous diversity in the vir gene family, the members of which are variably expressed and encode proteins that are exported to the host cell surface for the purpose of evading the host adaptive immune response ${ }^{20}$. Of the 313 vir genes included in the Salvador I reference assembly, less than a third are also observed in the four new assemblies (Fig. 3b). Unexpectedly, we encountered 15 'ultra-conserved' vir genes that were present in all assemblies and showed very low SNP diversity; in particular, one locus (PVX_113230) was invariant and showed the highest similarity (70\% protein sequence identity) of any vir to the homologous kir gene family in Plasmodium knowlesi, a zoonotic parasite (Fig. 3c). Unlike most vir genes, this locus also exhibits conserved synteny in more distantly related rodent malaria parasites. These attributes suggest that PVX_113230 is likely the founder of the vir family in the P. vivax lineage, and the lack of polymorphism suggests that the protein it encodes performs an ancestral role rather than host immune modulation. The molecular function of PVX_113230 could be related to erythrocyte invasion, as suggested by its distinct expression profile in erythrocytes relative to most vir genes ${ }^{21}$ (Supplementary Fig. 4).

This global census of genomic diversity in P. vivax has uncovered an unexpected degree of genetic polymorphism, much of which may

Figure 2 Neighbor-joining phylograms of $P$. vivax and $P$. falciparum constructed from presumably neutral SNPs occurring in fourfolddegenerate coding sites. Lineages are colored according to geographic origin. Branch lengths indicate considerable diversity in New World $P$. vivax strains and show no clear affiliation between New World and African $P$. vivax strains. Phylograms were constructed from 471,543 sites in $P$. vivax and 359,901 sites in $P$. falciparum. Numbers at nodes indicate percentages of bootstrap support. 
Figure 3 Diversity of $P$. vivax gene families. (a) Mean pairwise SNP diversity in $P$. vivax gene families. Gene families associated with merozoite invasion or immune response modulation (red text) show the highest diversity. Red bars on the box plots represent the $25^{\text {th }}$ to $75^{\text {th }}$ percentile range, and circles indicate outlier genes. (b) Limited overlapping vir repertoires of $P$. vivax isolates. vir genes exhibiting at least $70 \%$ sequence identity between isolates were included in the Venn diagram. A set of 15 ultra-conserved vir genes with more than $95 \%$ similarity in all comparisons are included in the central red circle. (c) A neighbor-joining phylogenetic tree of ultra-conserved vir genes and related paralogs from the vir 12 and vir 14 subfamilies. The most highly conserved vir, PVX_113230, has clear orthologs in other Plasmodium species.

translate into important functional variation. Our data stop short of suggesting the existence of distinct subspecies of $P$. vivax, similar to the subspecies $P$. vivax vivax and $P$. vivax hibernans proposed on the basis of relapse phenotype ${ }^{22}$. However, the extreme diversity we observe among these new reference strains suggests a more stable and older association of this parasite with humans than for P. falciparum, serving as a warning that the eradication of $P$. vivax could represent a qualitatively different task.

URLs. Malaria Research and Reference Reagent Resource Center (MR4): http://www.mr4.org/; TransposonPSI: http://transposonpsi. sourceforge.net/; $P$. vivax nuclear and apicoplast genome assemblies and gene calls and Sanger sequencing-based Dd2 assembly, http:// www.broadinstitute.org/annotation/genome/plasmodium_vivax/ MultiDownloads.html; PlasmoDB, http://plasmodb.org/.

\section{METHODS}

Methods and any associated references are available in the online version of the paper.

Accession codes. Illumina sequencing reads have been submitted to the NCBI Short Read Archive (SRA; accessions SRP007883, SRP007923, SRP000493 and SRP000316). SNP calls have been submitted to dbSNP and can also be downloaded from the Broad Institute website (see URLs). P. vivax nuclear and apicoplast genome assemblies and gene calls may be downloaded from GenBank (accessions AFMK01000000, AFBK01000000, AFNI01000000, AFNJ01000000, JQ437257, JQ437258 and JQ437259) and the Broad Institute website (see URLs).

Note: Supplementary information is available in the online version of the paper.

\section{ACKNOWLEDGMENTS}

This work has been funded, in whole or in part, by federal funds from the US National Institute of Allergy and Infectious Diseases (NIAID), the US National Institutes of Health (NIH) and the US Department of Health and Human Services, under contracts HHSN266200400001C and HHSN2722009000018C. We gratefully acknowledge the Indian Council of Medical Research for financial support of the Malaria Parasite Bank at the National Institute of Malaria Research, New Delhi, and the NIMR Director for providing all facilities. We thank the NIAID/National Human Genome Research Institute (NHGRI) Eukaryotic Pathogens and Disease Vectors Working Group and the Broad Institute Genome Sequencing Platform for
Conserved
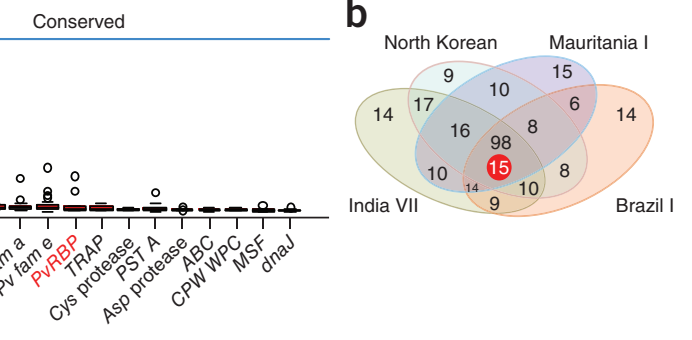

- 0

\section{(1)}

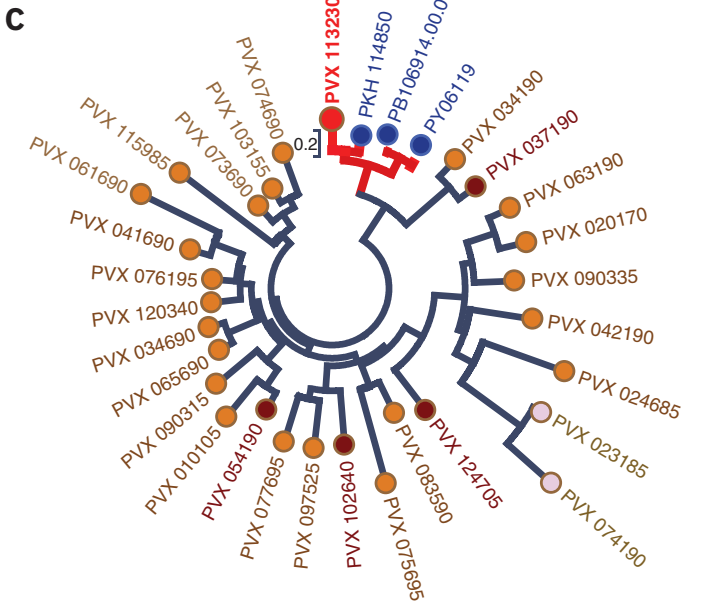
$\hat{c}^{-19}$

\section{0}

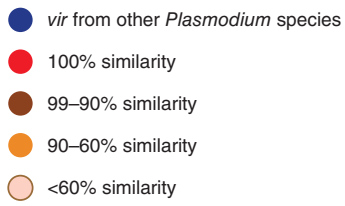

significant contributions to this project. K.G. and L.Y. are supported by a Global Health Program grant from the Bill and Melinda Gates Foundation. A.A.E. is supported by a grant from the NIH (RO1GM084320), and J.M.C. and P.L.S. are supported by grant U19AI089676, an NIAID International Center of Excellence for Malaria Research. The content is soley the responsibility of the authors and does not necessarily represent the official views of the NIH.

\section{AUTHOR CONTRIBUTIONS}

J.W.B. provided P. vivax strains, and A.R.A. and A.P.D. provided Indian P. falciparum material. S.M.S., S.S. and S.Y. performed genome assembly. S.G., J.M.G. and Q.Z. performed genome annotation. K.G., R.H.Y.J., L.Y. and D.E.N. performed analyses. S.B.C. performed project management. P.L.S. undertook experimental validation. D.E.N., A.A.E., B.W.B. and J.M.C. directed the analyses. D.E.N., A.A.E., J.W.B. and J.M.C. wrote the manuscript.

\section{COMPETING FINANCIAL INTERESTS}

The authors declare no competing financial interests.

Published online at http://www.nature.com/doifinder/10.1038/ng.2373.

Reprints and permissions information is available online at http://www.nature.com/ reprints/index.html.

1. Guerra, C.A. et al. The international limits and population at risk of Plasmodium vivax transmission in 2009. PLoS Negl. Trop. Dis. 4, e774 (2010).

2. Dobson, M.J. Malaria in England: a geographical and historical perspective. Parassitologia 36, 35-60 (1994).

3. Price, R.N., Douglas, N.M. \& Anstey, N.M. New developments in Plasmodium vivax malaria: severe disease and the rise of chloroquine resistance. Curr. Opin. Infect. Dis. 22, 430-435 (2009).

4. Carlton, J.M., Sina, B.J. \& Adams, J.H. Why is Plasmodium vivax a neglected tropical disease? PLoS Negl. Trop. Dis. 5, el160 (2011).

5. Winzeler, E.A. Malaria research in the post-genomic era. Nature 455, 751-756 (2008).

6. Carlton, J.M. et al. Comparative genomics of the neglected human malaria parasite Plasmodium vivax. Nature 455, 757-763 (2008).

7. Dharia, N.V. et al. Whole-genome sequencing and microarray analysis of ex vivo Plasmodium vivax reveal selective pressure on putative drug resistance genes. Proc. Natl. Acad. Sci. USA 107, 20045-20050 (2010).

8. Gnerre, S. et al. High-quality draft assemblies of mammalian genomes from massively parallel sequence data. Proc. Natl. Acad. Sci. USA 108, 1513-1518 (2011).

9. Feng, X. et al. Single-nucleotide polymorphisms and genome diversity in Plasmodium vivax. Proc. Natl. Acad. Sci. USA 100, 8502-8507 (2003). 
10. Mu, J. et al. Host switch leads to emergence of Plasmodium vivax malaria in humans. Mol. Biol. Evol. 22, 1686-1693 (2005).

11. DePristo, M.A. et al. A framework for variation discovery and genotyping using next-generation DNA sequencing data. Nat. Genet. 43, 491-498 (2011).

12. Gemayel, R., Vinces, M.D., Legendre, M. \& Verstrepen, K.J. Variable tandem repeats accelerate evolution of coding and regulatory sequences. Annu. Rev. Genet. 44, 445-477 (2010).

13. Joy, D.A., Mu, J., Jiang, H. \& Su, X. Genetic diversity and population history of Plasmodium falciparum and Plasmodium vivax. Parassitologia 48, 561-566 (2006).

14. Kumar, S. \& Subramanian, S. Mutation rates in mammalian genomes. Proc. Natl. Acad. Sci. USA 99, 803-808 (2002).

15. Paget-McNicol, S. \& Saul, A. Mutation rates in the dihydrofolate reductase gene of Plasmodium falciparum. Parasitology 122, 497-505 (2001).

16. Cornejo, O.E. \& Escalante, A.A. The origin and age of Plasmodium vivax. Trends Parasitol. 22, 558-563 (2006).

17. Conway, D.J. et al. Origin of Plasmodium falciparum malaria is traced by mitochondrial DNA. Mol. Biochem. Parasitol. 111, 163-171 (2000).

18. Carter, R. Speculations on the origins of Plasmodium vivax malaria. Trends Parasitol. 19, 214-219 (2003).

19. Thera, M.A. et al. A field trial to assess a blood-stage malaria vaccine. N. Engl. J. Med. 365, 1004-1013 (2011).

20. Fernandez-Becerra, c. et al. Plasmodium vivax and the importance of the subtelomeric multigene vir superfamily. Trends Parasitol. 25, 44-51 (2009).
21. Bozdech, Z. et al. The transcriptome of Plasmodium vivax reveals divergence and diversity of transcriptional regulation in malaria parasites. Proc. Natl. Acad. Sci. USA 105, 16290-16295 (2008).

22. Garnham, P.C. et al. A strain of Plasmodium vivax characterized by prolonged incubation: morphological and biological characteristics. Bull. World Health Organ. 52, 21-32 (1975).

23. Nayar, J.K. et al. Studies on a primaquine-tolerant strain of Plasmodium vivax from Brazil in Aotus and Saimiri monkeys. J. Parasitol. 83, 739-745 (1997).

24. Bhasin, V.K. \& Trager, W. Gametocyte-forming and non-gametocyte-forming clones of Plasmodium falciparum. Am. J. Trop. Med. Hyg. 33, 534-537 (1984).

25. Sullivan, J.S. et al. Adaptation of a strain of Plasmodium vivax from India to New World monkeys, chimpanzees, and anopheline mosquitoes. J. Parasitol. 87, 1398-1403 (2001).

26. Collins, W.E. et al. Studies on the North Korean strain of Plasmodium vivax in Aotus monkeys and different anophelines. J. Parasitol. 71, 20-27 (1985).

27. Wellems, T.E. et al. Chromosome size variation occurs in cloned Plasmodium falciparum on in vitro cultivation. Rev. Bras. Genet. 11, 813-825 (1988).

28. Collins, W.E. et al. Adaptation of a strain of Plasmodium vivax from Mauritania to New World monkeys and anopheline mosquitoes. J. Parasitol. 84, 619-621 (1998).

29. Gardner, M.J. et al. Genome sequence of the human malaria parasite Plasmodium falciparum. Nature 419, 498-511 (2002).

30. Melnikov, A. et al. Hybrid selection for sequencing pathogen genomes from clinical samples. Genome Biol. 12, R73 (2011). 


\section{ONLINE METHODS}

Parasite material. We chose four strains of $P$. vivax for whole-genome sequencing on the basis of geographic origin and phenotype to provide a resource of high-quality assembled and annotated sequences for the malaria research community. The North Korean strain has a long relapse phenotype that enables it to survive in the primate host through periods of drought and long winters when mosquito vectors for transmission are unavailable ${ }^{26}$. The Brazil I strain is highly resistant to the anti-relapse drug primaquine ${ }^{23}$. The India VII strain is the first Plasmodium species to be sequenced from India. The Mauritania I strain is a rare example of an African P. vivax strain that occurs among west Africans with Berber and Arab genetic backgrounds ${ }^{28}$. Genomic DNA for $P$. vivax sequencing was obtained from the leukocyte-depleted blood of infected, splenectomized Saimiri monkeys as described previously ${ }^{6}$. Genomewide fragment analysis of 15 microsatellites before sequencing confirmed their independent origins. DNA and frozen stabilates are available upon request at MR4 (see URLs).

Genome sequencing, assembly and annotation. Genomic DNA was used to construct two Illumina sequencing libraries for each $P$. vivax isolate, with library insert sizes of $180 \mathrm{bp}$ and $3 \mathrm{~kb}$. Each library was sequenced to a depth of at least 150 -fold coverage (to account for contaminating monkey host DNA) using 76-bp paired-end Illumina reads on an Illumina Genome Analyzer IIx sequencer. After filtering out reads with sequence similarity to monkey or primate sequence, each genome was assembled using the ALLPATHS-LG algorithm $^{8}$. Assembly quality was quantified using the N50 statistic for contigs and scaffolds, which describes the minimum contig or scaffold size such that the sum of the lengths of all contigs or scaffolds of equal or greater size accounts for at least half of the total assembly length. Synteny with the P. vivax Salvador I reference assembly was quantified for each de novo assembly by evaluating the concordance of approximately 60,000 randomly chosen pairs of 100-bp sequences, each with $100 \mathrm{~kb}$ of intervening sequence in the new assemblies. This method identifies breaks in synteny when the sequence pairs do not map to locations separated by $100 \mathrm{~kb}$ in the reference assembly or when only one member of a sequence pair is successfully mapped to the reference. Finally, for three of the four P. vivax isolates (North Korean, Mauritania I and Brazil I), we were able to recover a scaffold representing the complete apicoplast genome.

$P$. falciparum analyses were based on comparable Illumina read data generated from a single library for each isolate (insert size of $180 \mathrm{bp}$ ). Coverage for each of the isolates was as follows: Indian isolate 87_239, 42×; Indochina isolate Dd2, 196×; Honduran isolate HB3, 30×; Indian isolate ML-14, 41×; Senegalese isolate Th231.08, 59x.

The protein-coding genes in the $P$. vivax nuclear genomes were annotated using a combination of reference gene mapping, homology-based gene models (GeneWise) ${ }^{31}$, EST-based gene models and $a b$ initio gene predictions. Ribosomal RNAs (rRNAs) were identified with RNAmmer ${ }^{32}$. The tRNA features were identified using tRNAScanSE ${ }^{33}$. Other common RNA features were identified with RFAM ${ }^{34}$.

The MUMmer algorithm ${ }^{35}$ was used to align draft assemblies to the $P$. vivax Salvador I reference genome assembly. Neighboring syntenic alignment blocks were joined to form longer alignments, which were then used to map the gene coordinates from the reference genome to the draft assemblies. Homology-based gene models were created using tblastn to search against the draft genome assemblies with the UniRef90 protein database, a Plasmodium protein database created from the annotated proteins of P. falciparum 3D7 and P. vivax Salvador I. The resultant BLAST hits were used to create GeneWise gene models. Gene models were also built using 31,777 P. vivax ESTs available on GenBank, with an ORF cutoff length of $300 \mathrm{bp}$ (EST ORFs). Ab initio gene models were predicted using self-training GeneMark-ES ${ }^{36}$ and GeneId ${ }^{37}$ with parameters trained on genes from the $P$. vivax Salvador I genome.

The final merged gene set for each of the four sequenced $P$. vivax strains was created using the following workflow. If a mapped reference gene in a given assembly had intact start and stop codons, no frameshift or in-frame stop and no exons in the contig gaps, then the mapped reference gene was used directly as the gene model. If a mapped gene had a frameshift or in-frame stop, then the corresponding GeneMarkES gene model was selected. If a GeneWise gene model had no overlap with gene models from the previous two sources but had a non-generic gene product name or overlap with non-repeat PFAM domains, then the GeneWise feature was added as a gene model. Finally, if an EST ORF was at least $600 \mathrm{bp}$ in length and exhibited no overlap with models identified from the previous three sources, then the EST ORF was added as a gene model. The initial gene set was compared against tRNA and rRNA features, and genes were filtered out where appropriate. Additional gene filtering was performed by removing genes with $30 \%$ or more coding sequence overlap with hits in TransposonPSI (see URLs) $\left(e<1 \times 10^{-10}\right)$.

SNP calling. Sequencing reads from each isolate were aligned using the Burrows-Wheeler Aligner (BWA) ${ }^{38}$ to the reference assemblies of $P$. falciparum 3D7 (build v7.1) and P. vivax Salvador I (build v7.0), both downloaded from PlasmoDB (see URLs). SNPs were called using the Unified Genotyper ${ }^{11}$ in the GATK package ${ }^{39}$. SNPs with an estimated PHRED-style quality score of Q30 or greater were used for diversity analyses.

Microsatellite variants and validation. The mreps program ${ }^{40}$ was used to identify microsatellites in the reference assemblies of $P$. falciparum and $P$. vivax. For the microsatellite search, the mreps parameters were set as $\operatorname{minp}=1$ and $\operatorname{maxp}=9$. Searching under these conditions identified 538,794 and 95,990 microsatellite loci in P. falciparum and $P$. vivax, respectively. For $P$. falciparum, the allow-small parameter was employed to allow identification of small microsatellites that the mreps algorithm might otherwise flag as biologically insignificant due to the high AT content $(\sim 80 \%)$ of that genome.

Illumina sequencing reads were mapped to the $P$. falciparum $3 \mathrm{D} 7$ or $P$. vivax reference assemblies using $\mathrm{BWA}^{38}$ with the $-\mathrm{q} 5$ and -132 options. Base-quality score recalibration and local realignment around microsatellites ${ }^{11}$ were applied using GATK ${ }^{39}$. Custom Python scripts were used to filter out reads that did not span the entirety of a microsatellite interval in the genome. A final local realignment around microsatellite sequences, and insertion and/or deletion (indel) calling with standard hard filtering parameters was applied using GATK; accepted indels were converted into microsatellite-length genotypes. Illumina-based microsatellite calls were validated by comparison with calls made by aligning the Sanger sequence-based Dd2 assembly (see URLs) to the $3 \mathrm{D} 7$ reference assembly to generate a truth set of indels for genotyped microsatellites. Comparison of the Illumina-based calls to the Sanger sequencingbased calls indicated that our Illumina-based calling method exhibits a specificity of $100 \%$ and a sensitivity of $97 \%$, on the basis of 81,569 AT dinucleotide microsatellites callable in both data sets. $P$ values for evaluating the significance of the difference in microsatellite diversity between species were generated by resampling $\pi$ values for each species 10,000 times and noting the frequency with which a difference in mean $\pi$ occurred that was equal to or greater than the observed difference in mean $\pi$. The overall comparison of microsatellite diversity across motif unit sizes was performed using only microsatellites with eight or more repeat units, given that, as previously observed for P. vivax ${ }^{41}$, these longer microsatellites were observed to be more polymorphic in both species (Supplementary Fig. 5).

Evolutionary analyses and TMRCA calculation. Mean pairwise diversity at all nucleotide sites $(\pi)$, synonymous sites $\left(\pi_{\mathrm{S}}\right)$ and nonsynonymous sites $\left(\pi_{\mathrm{N}}\right)$ was calculated for each gene in the reference annotations for $P$. falciparum and $P$. vivax using the method described in Table 1 of ref. 42. P. knowlesi orthologs were employed to calculate interspecies $\mathrm{d}_{\mathrm{N}} / \mathrm{d}_{\mathrm{S}}$ ratios using PAML v4.5 (ref. 43) with sequences from the Salvador I isolate of P. vivax. A matrix of pairwise nucleotide distances between isolates (Supplementary Table 6) was constructed for each species using SNPs identified in fourfold-degenerate synonymous coding sites of genes with orthologs in each species. To control for differences in the nucleotide substitution profile between species and enable direct comparison of branch lengths, pairwise distances were normalized by empirical nucleotide transition matrices (Supplementary Table 7) constructed for each species by rooting fourfold-degenerate polymorphisms using an outgroup species ( $P$. knowlesi for $P$. vivax and $P$. reichenowi for $P$. falciparum). Phylograms were constructed from pairwise distance matrices using the Neighbor-Joining method. The lower bound of the TMRCA was estimated for each species as the deepest pairwise divergence. SNP functional enrichment analyses were carried out using Mann-Whitney $U$ tests, and $Z$ scores were interpreted using a Bonferroni correction for multiple testing. 
31. Birney, E., Clamp, M. \& Durbin, R. GeneWise and Genomewise. Genome Res. 14, 988-995 (2004).

32. Lagesen, K. et al. RNAmmer: consistent and rapid annotation of ribosomal RNA genes. Nucleic Acids Res. 35, 3100-3108 (2007).

33. Lowe, T.M. \& Eddy, S.R. tRNAscan-SE: a program for improved detection of transfer RNA genes in genomic sequence. Nucleic Acids Res. 25, 955-964 (1997).

34. Griffiths-Jones, S. Annotating non-coding RNAs with Rfam. Curr. Protoc. Bioinformatics Chapter 12, Unit 12.5 (2005).

35. Kurtz, S. et al. Versatile and open software for comparing large genomes. Genome Biol. 5, R12 (2004).

36. Ter-Hovhannisyan, V., Lomsadze, A., Chernoff, Y.O. \& Borodovsky, M. Gene prediction in novel fungal genomes using an ab initio algorithm with unsupervised training. Genome Res. 18, 1979-1990 (2008).
37. Blanco, E. \& Abril, J.F. Computational gene annotation in new genome assemblies using GenelD. Methods Mol. Biol. 537, 243-261 (2009).

38. Li, H. \& Durbin, R. Fast and accurate short read alignment with Burrows-Wheeler transform. Bioinformatics 25, 1754-1760 (2009).

39. McKenna, A. et al. The Genome Analysis Toolkit: a MapReduce framework for analyzing next-generation DNA sequencing data. Genome Res. 20, 1297-1303 (2010).

40. Kolpakov, R., Bana, G. \& Kucherov, G. mreps: efficient and flexible detection of tandem repeats in DNA. Nucleic Acids Res. 31, 3672-3678 (2003).

41. Russell, B., Suwanarusk, R. \& Lek-Uthai, U. Plasmodium vivax genetic diversity: microsatellite length matters. Trends Parasitol. 22, 399-401 (2006).

42. Ina, Y. New methods for estimating the numbers of synonymous and nonsynonymous substitutions. J. Mol. Evol. 40, 190-226 (1995).

43. Yang, Z. PAML 4: phylogenetic analysis by maximum likelihood. Mol. Biol. Evol. 24 1586-1591 (2007). 